\title{
Analgesia Controlada pelo Paciente com Fentanil e Sufentanil no Pós-Operatório de Reconstrução de Ligamentos do Joelho: Estudo Comparativo
}

\author{
Patient Controlled Analgesia with Fentanyl or Sufentanil in the Post- \\ operative Period of Knee Ligament Reconstruction: Comparative Study
}

Marcelo Negrão Lutti, TSA ${ }^{1}$, João Lopes Vieira, TSA ${ }^{2}$, Dante Roberto Eickhoff ${ }^{3}$,

Daniel de Carli ${ }^{3}$, Marcelo Antônio de Carvalho ${ }^{3}$

\begin{abstract}
RESUMO
Lutti MN, Vieira JL, Eickhoff DR, Carli D, Carvalho MA - Analgesia Controlada pelo Paciente com Fentanil ou Sufentanil no Pós-Operatório de Reconstrução de Ligamentos do Joelho: Estudo Comparativo
\end{abstract}

Justificativa e Objetivos - Os opióides têm sido utilizados por via peridural associados ou não a anestésicos locais para analgesia pós-operatória de forma contínua elou em bolus controlado pelo paciente. O objetivo deste estudo foi comparar a analgesia pós-operatória entre o fentanil e sufentanil em infusão contínua e em bolus por via peridural, em pacientes submetidos à reconstrução de ligamento do joelho.

Método - Participaram do estudo 70 pacientes com idades entre 16 e 47 anos, estado físico ASA I e II, divididos aleatoriamente em dois grupos: Grupo $F$ (fentanil) e Grupo $S$ (sufentanil). Todos os pacientes foram submetidos à anestesia peridural com bupivacaína a 0,5\% (100 mg) com epinefrina 1:200.000 associada a fentanil ( $100 \mathrm{mg})$. Ao final da cirurgia, os pacientes receberam fentanil (Grupo F) ou sufentanil (Grupo S) por via peridural em regime de infusão contínua mais bolus liberados pelo paciente.. No Grupo $F$ foi utilizada solução fisiológica $(85 \mathrm{ml})$ contendo fentanil $500 \mu \mathrm{g}(10 \mathrm{ml})$ e bupivacaína (5 $\mathrm{ml}$ a $0,5 \%)$. No Grupo $S$ foi utilizada solução fisiológica (92 ml) contendo sufentanil $150 \mu \mathrm{g}(3 \mathrm{ml})$ e bupivacaína (5 $\mathrm{ml}$ a $0,5 \%)$. Para os dois grupos a bomba de infusão foi programada inicialmente em $5 \mathrm{ml} . \mathrm{h}^{-1}$, com dose de 2 $\mathrm{ml}$ em bolus liberado pelo paciente num intervalo de 15 minutos. Foram comparados os seguintes parâmetros: dor, número de bolus acionados, consumo de opióides, bloqueio motor, sedação e efeitos colaterais.

Resultados - Não houve diferença entre os grupos quanto à qualidade da analgesia, sendo a maioria de boa qualidade (EAV 0 a 2). Houve diferença quanto ao número de bolus liberados. No Grupo F solicitou mais bolus que o Grupo S. Não houve diferença quanto ao volume total e tempo de infusão total. Não houve bloqueio motor após a instituição da analgesia controlada pelo paciente. A incidência de vômitos e retenção urinária foi maior no Grupo S e quanto à sedação e ao prurido, não houve diferença entre os grupos.

\footnotetext{
* Recebido do (Received from) CET/SBA do Instituto Penido Burnier, Campinas, $S P$

1. Instrutor do CET/SBA

2. Co-responsável pelo CET/SBA

3. EX-ME do CET/SBA

Apresentado (Submitted) em 26 de junho de 2001

Aceito (Accepted) para publicação em 10 de outubro de 2001

Correspondência para (Mail to):

Dr. Marcelo Negrão Lutti

R. Dr. José Ferreira de Camargo, 1280 - Jardim Planalto

13092-001 Campinas, SP

(C) Sociedade Brasileira de Anestesiologia, 2002
}

Conclusões - O fentanil ou o sufentanil contínuos em bolus acionados pelo paciente, por via peridural, nas doses utilizadas neste estudo, apresentaram excelente analgesia pós-operatória. No entanto, o sufentanil apresentou efeitos colaterais mais intensos que o fentanil.

UNITERMOS: ANALGESIA, Pós-operatória: analgesia controlada pelo paciente; ANALGÉSICOS, Opióides: fentanil, sufentanil; TÉCNICAS ANESTÉSICAS, Regional: peridural contínua

\section{SUMMARY}

Lutti MN, Vieira JL, Eickhoff DR, Carli D, Carvalho MA - Patient Controlled Analgesia with Fentanyl or Sufentanil in the Postoperative Period of Knee Ligament Reconstruction: Comparative Study

Background and Objectives - Epidural opioids, associated or not to local anesthetics, have been used for postoperative analgesia in continuous infusion and/or patient controlled boluses. The aim of this study was to compare postoperative analgesia provided by epidural fentanyl or sufentanil, in bolus or continuous infusion, in patients submitted to knee ligament reconstruction.

Methods - Seventy ASA I - II patients, aged 16 to 47 years, were randomly distributed in two groups. All patients were submitted to epidural anesthesia with $0.5 \%$ bupivacaine $(100 \mathrm{mg})$ with epinephrine 1:200,000 associated to fentanyl $(100 \mathrm{mg})$. At the end of the procedure, patients received epidural fentanyl (Group F) or sufentanil (Group S) in continuous infusion plus patient controlled boluses. Group Finfusion solution was made of saline (85 ml), $500 \mu \mathrm{g}$ fentanyl $(10 \mathrm{ml})$ and $0.5 \%$ bupivacaine $(5 \mathrm{ml})$. Group $S$ solution was made of saline $(92 \mathrm{ml}), 150 \mu \mathrm{g}$ sufentanil $(3 \mathrm{ml})$ and $0.5 \%$ plain bupivacaine $(5 \mathrm{ml})$. Infusion pump's flow was initially programmed to $5 \mathrm{ml} . \mathrm{h}^{-1}$, with $2 \mathrm{ml}$ patient controlled bolus doses every 15 minutes at most, for both groups. The following parameters were compared: pain, number of patient controlled boluses, opioid consumption, motor block, sedation and side-effects.

Results - There have been no statistically significant difference in analgesia quality between groups, being in most cases rated good (AVS 0 to 2). There has been a significant difference in the number of patient controlled boluses, with Group F needing more boluses than Group S. There has been no difference in total infused solution volume and total infusion time. There has been no motor block after beginning of patient-controlled analgesia. Vomiting and urine retention incidences were higher in Group $S$ and there have been no significant differences in sedation and pruritus between groups.

Conclusions - Epidural fentanyl or sufentanil in continuous epidural infusion and patient-controlled boluses in the doses used in this study have induced excellent postoperative analgesia. However, sufentanil caused more severe side effects than fentanyl.

KEYWORDS - ANALGESIA, Postoperative: patient controlled analgesia; ANALGESICS, Opioids: fentanyl, sufentanil; ANESTHETIC TECHNIQUES, Regional: continuous epidural 


\section{INTRODUÇÃO}

$\mathrm{O}$ desenvolvimento de novas técnicas de analgesia pós-operatória possibilitou planejamentos anestésicos visando um pós-operatório sem dor com recuperação e alta precoce dos pacientes ${ }^{1}$. Assim, com a utilização de anestésicos de longa duração associados ou não aos opióides em bloqueios periféricos, bloqueio peridural e subaracnóideo contínuos, na dependência do tipo de cirurgia, é possível melhorar a qualidade da analgesia pós-operatória ${ }^{2-6}$.

Com a associação de opióides aos anestésicos locais de longa duração, ocorre uma melhora da qualidade do bloqueio peridural e subaracnóideo, possibilitando, nos casos em que há associação da peridural com anestesia geral, a manutenção de um plano anestésico mais superficial, com menor consumo de drogas e anestésicos inalatórios ${ }^{5-7}$.

Tem-se utilizado fentanil, ou morfina, associados aos anestésicos locais, por via peridural em dose única ou através de cateter, em doses intermitentes ou contínuas por bomba de infusão programada ${ }^{8}$. Estas bombas permitem o controle da analgesia pelo paciente, trazendo mais conforto e segurança, possibilitando uma maior interatividade do doente com sua recuperação ${ }^{8-14}$. Outra utilização destas bombas se faz por via venosa, porém com maior consumo de opióides ${ }^{5,6}$.

Os efeitos colaterais destas técnicas, utilizando opióides, incluem sedação, náuseas, vômitos, prurido e retenção urinária. Outro efeito mais grave é a depressão respiratória, causada geralmente pela morfina ${ }^{5,6,21-24}$.

A cirurgia de reconstrução do ligamento cruzado anterior do joelho, com transposição do tendão patelar, por via artroscópica, é muito dolorosa especialmente no primeiro dia de pós-operatório, aumentando o tempo de permanência hospitalar e dificultando a instituição de fisioterapia precoce.

Estudo recente ${ }^{8}$ mostrou a eficácia do emprego do fentanil ou morfina associados à baixa concentração de bupivacaína, em infusão contínua, em regime de analgesia controlada pelo paciente (ACP), no controle da dor pós-operatória. Aeficácia do método permitiu fisioterapia precoce e alta dos pacientes em 24 horas $^{8}$. Demonstrou também a baixa incidência de efeitos colaterais (sedação, náusea, vômito e prurido) sendo menor para o fentanil ${ }^{8}$. Além do mais, as doses de infusão contínua e em bolus foram menores do que aquelas administradas por outros autores ${ }^{8,15-17}$.

O sufentanil também tem sido empregado em ACP mostrando bons resultados no controle da dor pós-operatória ${ }^{18,19}$. O objetivo deste estudo foi comparar o fentanil e o sufentanil em analgesia controlada pelo paciente, por via peridural, com bomba de infusão contínua, em pacientes submetidos a cirurgias para reconstrução de ligamentos de joelho. Estudou-se, ainda, o consumo de opióides e os efeitos colaterais como sedação, prurido, retenção urinária e depressão respiratória.

\section{MÉTODO}

Após a aprovação do protocolo desta pesquisa pela Comissão de Ética do Centro Médico de Campinas e consentimen- to formal, participaram do estudo 70 pacientes com idades entre 16 e 47 anos, estado físico ASA I e II, submetidos à cirurgia de reconstrução de ligamentos do joelho sob anestesia peridural.

Todos os pacientes receberam, como medicação pré-anestésica, diazepam (10 mg) por via oral 60 minutos antes da cirurgia.

$\mathrm{Na}$ sala de cirurgia foi feita venopunção com cateter 18 ou $16 \mathrm{G}$ em veia de membro superior e infusão de solução de Ringer com lactato $(500 \mathrm{ml})$. A monitorização constou de cardioscópio na derivação $D_{\|}$, oximetria de pulso, medida da pressão arterial por método automático não invasivo. Antes do bloqueio peridural os pacientes receberam, por via venosa, sedação leve com frações tituladas da mistura de midazolam ( $5 \mathrm{mg}$ ) e fentanil $(50 \mu \mathrm{g})$ em doses suficientes para se obter um paciente calmo e cooperativo. Aseguir, foi realizada a punção peridural na região lombar, com agulha de Tuohy 16G. Após dose-teste negativa com lidocaína a $2 \%$ com epinefrina 1:200.000 (60 mg), procedeu-se à injeção de bupivacaína a 0,5\% (100 mg) com epinefrina $1: 200.000$, associada a fentanil $(100 \mu \mathrm{g})$. Em seguida, foi colocado um cateter no espaço peridural em direção cefálica com a finalidade de realizar analgesia pós-operatória controlada pelo paciente com bomba de infusão. Terminado o ato operatório foi instalada a bomba de infusão no cateter peridural.

No pós-operatório imediato os pacientes foram divididos aleatoriamente em dois grupos: Grupo $F(n=35)$ e Grupo $S$ ( $n=$ 35 ), nos quais foram colocadas bombas de infusão com as soluções e respectivas programações apresentadas nas tabelas I e II.

Nos casos de maior ou menor necessidade de analgesia, a dose da solução seria reprogramada para mais ou para menos $20 \%$ da dose inicial. Ao aparecimento de efeitos indesejáveis, as doses também seriam reprogramadas para menos $20 \%$ da dose inicial. Persistindo os efeitos indesejáveis, estes seriam tratados da seguinte maneira: para os casos de prurido intenso - 1) difenidramina (50 mg), por via muscular; 2) naloxona $2 \mathrm{ml}+8 \mathrm{ml}$ de água destilada - titulando-se a dose por via venosa. Os casos de depressão respiratória com freqüência respiratória igual ou menor que 9 i.p.m. seriam tratados com naloxona $2 \mathrm{ml}+8 \mathrm{ml}$ de água destilada titulando-se a dose por via venosa. Para os casos de náuseas e vômitos seria administrada metoclopramida (10 mg) por via venosa.

Tabela I - Grupo F - Solução Contendo Fentanil ${ }^{8}$

\begin{tabular}{ll}
\hline Composição da Solução & \\
Solução fisiológica & $85 \mathrm{ml}$ \\
Bupivacaína a $0,5 \%$ & $5 \mathrm{ml}(25 \mathrm{mg})$ \\
Fentanil & $10 \mathrm{ml}(500 \mu \mathrm{g})$ \\
Programação da Infusão & \\
Infusão contínua & $5 \mathrm{ml} \cdot \mathrm{h}^{-1}$ \\
Liberação em bolus & $2 \mathrm{ml}$, com intervalos de 15 minutos \\
\hline
\end{tabular}


Tabela II - Grupo S - Solução Contendo Sufentanil

\begin{tabular}{ll}
\hline Composição da Solução & \\
Solução fisiológica & $92 \mathrm{ml}$ \\
Bupivacaína a $0,5 \%$ & $5 \mathrm{ml}(25 \mathrm{mg})$ \\
Sufentanil & $3 \mathrm{ml}(150 \mu \mathrm{g})$ \\
Programação da Infusão & \\
Infusão contínua & $5 \mathrm{ml} \cdot \mathrm{h}^{-1}$ \\
Liberação em bolus & $2 \mathrm{ml}$, com intervalos de 15 minutos \\
\hline
\end{tabular}

O grau de sedação foi avaliado através dos seguintes parâmetros: 0 = nenhuma sedação; 1 = sedação leve (ocasionalmente sonolento, desperta ao ser chamado); 2 = sedação moderada (freqüentemente sonolento, desperta ao ser chamado; 3 = sedação intensa (sonolento, desperta mediante estímulo doloroso). A avaliação do bloqueio motor foi realizada pelo método de Bromage: 0 = ausência de bloqueio motor, 1 = incapacidade para levantar as pernas estendidas, 2 = incapacidade para flexionar os joelhos, 3 = incapacidade para flexionar os pés.

A analgesia foi avaliada através da Escala Analógica Visual (EAV) de 0 a 10, em que 0 seria nenhuma dor e 10 a pior dor possível. Aanalgesia foi avaliada dentro de dois períodos: 12 horas após cirurgia e a partir daí até a alta do paciente. Foram medidos: o volume da solução infundida, a dose do opióide utilizado, o tempo de infusão da solução, o número de bolus solicitados e número de bolus efetivamente liberados ao paciente. Foram anotados os efeitos colaterais como náuseas, vômitos, prurido, retenção urinária, sedação e depressão respiratória.

O teste $t$ Para Dois Grupos Independentes foi utilizado para análise estatística da idade, altura, peso, náuseas, vômitos, volume total de infusão na peridural pela bomba de ACP, número de bolus solicitados, número de bolus liberados e o tempo de infusão das soluções. A prova não paramétrica de Mann-Whitney foi utilizada para análise estatística do estado físico (ASA), sexo, prurido, sedação, retenção urinária e Escala Analógica Visual para dor. Foi estabelecido valor de $p$ $<0,05$.

\section{RESULTADOS}

Não houve diferença significativa entre os grupos com relação a idade, peso, altura, sexo e estado físico (Tabela III). Não há evidências de que os tratamentos sejam diferentes quanto à dor observada na Escala Analógica Visual (EAV) nas primeiras 12 horas e nas horas seguintes até a alta dos pacientes, quanto ao número de bolus liberados; entretanto, quanto à solicitação de bolus houve diferença significativa; o Grupo F - (Fentanil) solicitou mais bolus (Tabela IV).

Se considerarmos que os escores 0 (ausência de dor), 1 e 2 (dor leve-tolerável), houve boa resposta ao tratamento da dor pós-operatória em ambos os grupos (Tabelas $\mathrm{V}$ e VI). Não houve caso de dor insuportável em nenhum grupo, apenas dois casos no grupo $\mathrm{F}$ a dor atingiu o escore 8 .
Tabela III - Dados Demográficos

\begin{tabular}{lccccc}
\hline & $\begin{array}{c}\text { Idade * } \\
(\mathrm{anos})\end{array}$ & $\begin{array}{c}\text { Peso * } \\
(\mathrm{kg})\end{array}$ & $\begin{array}{c}\text { Altura * } \\
(\mathrm{cm})\end{array}$ & $\begin{array}{c}\text { Sexo } \\
(\mathrm{M} / \mathrm{F})\end{array}$ & $\begin{array}{c}\text { ASA } \\
\mathrm{l} / \mathrm{II}\end{array}$ \\
\hline $\begin{array}{l}\text { Grupo } \mathrm{F} \\
(\mathrm{n}=35)\end{array}$ & $28,40 \pm 8,74$ & $79,77 \pm 14,84$ & $175,49 \pm 8,05$ & $33 / 2$ & $26 / 9$ \\
$\begin{array}{l}\text { Grupo } S \\
(\mathrm{n}=35)\end{array}$ & $28,51 \pm 7,69$ & $81,85 \pm 12,21$ & $178,91 \pm 6,95$ & $33 / 2$ & $30 / 5$ \\
\hline * Valores expressos pela Média $\pm \mathrm{DP}$ & & & & \\
\hline
\end{tabular}

Tabela IV - Número de Bolus Solicitado, Número de Bolus Liberados, Volume Total da Solução Infundida e Tempo de Infusão (Média \pm DP)

\begin{tabular}{lcc}
\hline Eventos & $\begin{array}{c}\text { Grupo } \mathrm{F} \\
(\mathrm{n}=35)\end{array}$ & $\begin{array}{c}\text { Grupo S } \\
(\mathrm{n}=35)\end{array}$ \\
\hline $\mathrm{N}^{\circ}$ bolus solicitados & $7,77 \pm 7,10^{*}$ & $4,51 \pm 4,93^{*}$ \\
$\mathrm{~N}^{\circ}$ bolus liberados & $4,54 \pm 2,94$ & $3,09 \pm 2,93$ \\
Volume total infusão $(\mathrm{ml})$ & $78,97 \pm 17,35$ & $87,00 \pm 18,64$ \\
Tempo de infusão (hora) & $17,10 \pm 0,16$ & $18,25 \pm 0,16$ \\
\hline
\end{tabular}

* Diferença estatística $(p<0,05)$

Tabela V - Escala Analógica Visual (EAV) Aplicada a Partir da $13^{a}$ Hora até a Alta dos Pacientes.

\begin{tabular}{lcc}
\hline Escores & $\begin{array}{c}\text { Grupo } F \\
(\mathrm{n}=35)\end{array}$ & $\begin{array}{c}\text { Grupo S } \\
(\mathrm{n}=35)\end{array}$ \\
\hline 0 & 25 & 17 \\
1 & 5 & 7 \\
2 & 3 & 2 \\
3 & 0 & 4 \\
4 & 1 & 1 \\
5 & 1 & 2 \\
6 & 0 & 0 \\
7 & 0 & 2 \\
8 & 0 & 0 \\
9 & 0 & 0 \\
10 & 0 & 0 \\
\hline
\end{tabular}

Tabela VI - Escala Analógica Visual (EAV) Aplicada nas Primeiras 12 Horas de Pós-Operatório

\begin{tabular}{lcc}
\hline Escores & $\begin{array}{c}\text { Grupo } F \\
(\mathrm{n}=35)\end{array}$ & $\begin{array}{c}\text { Grupo S } \\
(\mathrm{n}=35)\end{array}$ \\
\hline 0 & 11 & 13 \\
1 & 8 & 6 \\
2 & 7 & 4 \\
3 & 2 & 5 \\
4 & 1 & 3 \\
5 & 3 & 4 \\
6 & 0 & 0 \\
7 & 1 & 0 \\
8 & 2 & 0 \\
9 & 0 & 0 \\
10 & 0 & 0 \\
\hline
\end{tabular}

A retenção urinária ocorreu em 5 casos no grupo sufentanil, sendo necessária sondagem vesical (Tabela VII).

Revista Brasileira de Anestesiologia Vol. 52, № 2, Março - Abril, 2002 
Tabela VII - Necessidade de Sondagem Vesical no Pós-Operatório.

\begin{tabular}{lcc}
\hline$N^{\circ}$ de Sondagem & $\begin{array}{c}\text { Grupo } F \\
(\mathrm{n}=35)\end{array}$ & $\begin{array}{c}\text { Grupo } S \\
(\mathrm{n}=35)\end{array}$ \\
\hline 0 & 35 & 30 \\
1 & 0 & 5 \\
\hline
\end{tabular}

A sedação recebeu grau zero na maioria dos pacientes em ambos os grupos (34 cada) sem diferença estatisticamente significante. Foram registrados dois casos de sedação leve (um em cada grupo) (Tabela VIII). Da mesma forma foi o comportamento quanto à presença de prurido, sendo desnecessário o tratamento (Tabela IX).

Tabela VIII - Sedação dos Pacientes

\begin{tabular}{lcc}
\hline Escore & $\begin{array}{c}\text { Grupo } F \\
(\mathrm{n}=35)\end{array}$ & $\begin{array}{c}\text { Grupo S } \\
(\mathrm{n}=35)\end{array}$ \\
\hline 0 & 34 & 34 \\
1 & 1 & 1 \\
2 & 0 & 0 \\
\hline
\end{tabular}

Tabela IX - Prurido Apresentado pelos dos Pacientes

\begin{tabular}{lcc}
\hline Escore & $\begin{array}{c}\text { Grupo } F \\
(\mathrm{n}=35)\end{array}$ & $\begin{array}{c}\text { Grupo S } \\
(\mathrm{n}=35)\end{array}$ \\
\hline 0 (ausente) & 34 & 34 \\
1 (leve) & 1 & 1 \\
2 (moderado) & 0 & 0 \\
3 (intenso) & 0 & 0 \\
\hline
\end{tabular}

Apesar de baixa, houve incidência maior de vômitos no grupo sufentanil (Tabela $\mathrm{X}$ ).

Tabela X - Náuseas e Vômitos Durante as Infusões

\begin{tabular}{lcccc}
\hline Grupos & GF & GF $\%$ & GS & GS \% \\
\hline Náuseas & 2 & 5,71 & 3 & 8,57 \\
Vômitos & $1^{*}$ & $2,85^{*}$ & $2^{*}$ & $5,71^{*}$ \\
\hline
\end{tabular}

*Diferença estatística

Após a movimentação dos membros inferiores na sala de recuperação pós-anestésica não foi registrado mais nenhum caso de bloqueio motor com os regimes de infusão instituídos.

\section{DISCUSSÃO}

A reconstrução de ligamentos de joelho é um procedimento de alta incidência em indivíduos jovens, do sexo masculino, cujo pós-operatório é muito doloroso principalmente nas primeiras 24 horas, período em que é realizada a primeira mobilização do joelho operado com a finalidade de fisioterapia precoce.

De acordo com alguns autores ${ }^{1,8,25-28}$, a anestesia peridural com anestésico local de longa duração associada a um opióide e cateter peridural para analgesia pós-operatória é a meIhor escolha para este procedimento. O presente estudo utili-

Revista Brasileira de Anestesiologia

Vol. 52, № 2, Março - Abril, 2002 zou bupivacaína a 0,5\% com adrenalina 1:200.000 associada a fentanil $(100 \mu \mathrm{g})$ para prover analgesia durante o ato cirúrgico. Essa dose de fentanil utilizada no inicio da anestesia peridural não interferiu no resultado da analgesia pós-operatória devido à curta duração de seu efeito.

A instalação da bomba de ACP ocorreu antes do término do bloqueio anestésico com o paciente ainda na sala de cirurgia; entretanto, a alta do paciente da sala de recuperação pós-anestésica só foi autorizada após a recuperação total do bloqueio motor.

O fentanil é uma droga lipofílica e de maior potência analgésica que a morfina, que é hidrofílica; no entanto, alguns trabalhos apresentam uma equiparação analgésica entre as duas drogas ${ }^{2,6,8}$, porém com maiores efeitos colaterais da morfina (náuseas, vômitos e sedação). Nesta pesquisa, a comparação entre o fentanil e o sufentanil associados à bupivacaína em baixa concentração por via peridural foram administrados por bomba de ACP, mostrou analgesia eficiente no pós-operatório com o mínimo de efeitos colaterais possível. O resultado quanto à análise estatística da Escala Analógica Visual (EAV) mostrou que não houve diferença no tratamento da dor no pós-operatório até a alta dos pacientes; porém, dois pacientes do grupo do fentanil apresentaram escore 8 na EAV durante a movimentação, aumentando-se $20 \%$ a dose de infusão contínua do fentanil para estes dois pacientes. Quanto ao número de bolus liberados, o volume total e o tempo de infusão, não houve diferença significativa, mostrando a eficácia analgésica semelhante do fentanil com o sufentanil, nas doses utilizadas, no tratamento da dor pós-operatória.

Com a baixa concentração da bupivacaína $(0,025 \%)$, não houve nenhum caso de bloqueio motor, mostrando que há necessidade de concentrações maiores de bupivacaína para que o mesmo ocorra. Os pacientes mantiveram-se totalmente despertos no pós-operatório com apenas um paciente de cada grupo (GF e GS) apresentado grau 1 na escala de sedação, mostrando que houve segurança quanto ao efeito colateral mais temível, a depressão respiratória, com as doses utilizadas de ambos opióides. Poucos pacientes apresentaram náusea e vômitos em ambos os grupos ( $G F=3$ e GS=5), havendo diferença estatística significativa em favor do fentanil. Prurido leve foi relatado por apenas um paciente em cada grupo. Já a retenção urinária ocorreu em cinco pacientes do grupo do sufentanil, havendo necessidade de sondagem vesical de alívio, e nenhum caso ocorreu no grupo do fentanil.

A qualidade da analgesia e a baixa incidência de complicações mostram que tanto o fentanil como o sufentanil são meIhores do que a morfina para o regime de ACP para analgesia pós-operatória na cirurgia de reconstrução de ligamentos do joelho. Fato este comprovado quando se compara com outro estudo, realizado com o mesmo método, quando se comparou fentanil e morfina ${ }^{8}$.

Pode-se concluir que nas condições deste estudo a analgesia pós-operatória com infusão contínua através de bomba de ACP associada a bolus acionado pelo paciente por via peridural é um excelente método para tratamento da dor em pa- 
cientes que se submetem à reconstrução de ligamentos do joelho, principalmente quando se usam soluções de anestésicos locais de longa duração e baixa concentração, associados a opióides como o fentanil e sufentanil. Tanto o fentanil como o sufentanil foram eficientes quanto à analgesia. No entanto, o sufentanil apresentou efeitos colaterais um pouco mais intensos que o fentanil.

\section{Patient Controlled Analgesia with Fentanyl or Sufentanil in the Postopera- tive Period of Knee Ligament Recons- truction: Comparative Study}

Marcelo Negrão Lutti, TSA, M.D., João Lopes Vieira, TSA, M.D., Dante Roberto Eickhoff, M.D., Daniel de Carli, M.D., Marcelo Antônio de Carvalho, M.D.

\section{INTRODUCTION}

The development of new postoperative analgesic techniques has allowed for anesthetic planning aiming at a pain-free postoperative period with patients early recovery and discharge ${ }^{1}$. So, depending on the surgery, the use of long lasting anesthetics associated or not to opioids in peripheral, continuous epidural and spinal blocks, makes possible to improve postoperative analgesia ${ }^{2-6}$.

Opioids associated to long-lasting local anesthetics improve epidural and spinal block quality, allowing for the maintenance of a more superficial anesthesia with less drug and inhalational anesthetics consumption, when combined epidural/general anesthesia is used ${ }^{5-7}$.

Epidural fentanyl or morphine associated to local anesthetics have been used in single doses or through catheter's, in intermittent boluses or continuous infusion by programmed infusion pump's ${ }^{8}$.

These pumps allow analgesia to be patient-controlled, not only bringing more comfort and safety but also creating a higher interactivity of patients with their recovery ${ }^{8-14}$. There are pumps designed for intravenous infusion of drugs too, though its use is related to higher opioid consumption ${ }^{5,6}$. Side-effects of opioids infusion techniques are sedation, nausea, vomiting, pruritus and urine retention. Another more severe effect is respiratory depression, usually related to morphine analgesia ${ }^{5,6,21-24}$.

Knee anterior cruciate ligament reconstruction with arthroscopic patellar tendon transposition is very painful, especially during the first postoperative day. It increases time for hospital discharge and makes difficult early physiotherapy. A recent study ${ }^{8}$ has shown the efficacy of fentanyl or morphine associated to low bupivacaine concentrations in continuous infusion to control postoperative pain in a patient-controlled analgesia system (PCA), which allowed early physiotherapy and patients discharge in 24 hours $^{8}$. It has also shown a low incidence of side-effects (sedation, nausea, vomiting and pruritus), specially with fentanyl ${ }^{8}$. Moreover, continuous infusion and bolus doses were lower than those administered by other authors ${ }^{8,15-17}$

Sufentanil has also been used in PCA with good results in controlling postoperative pain ${ }^{18,19}$.

The aim of this study was to compare fentanyl and sufentanil in patient-controlled epidural analgesia with continuous infusion pump, in patients submitted to knee ligament reconstruction. Besides analgesia, other parameters were also evaluated, like opioid consumption and side-effects, such as sedation, pruritus, urine retention and respiratory depression.

\section{METHODS}

After the Centro Médico de Campinas Ethics Committee approval, 70 patients aged 16 to 47 years, physical status ASA I and II, scheduled to undergo knee ligament reconstruction under epidural anesthesia gave their written informed consent to be included in this study.

All patients were premedicated with oral diazepam $(10 \mathrm{mg})$ 60 minutes before surgery.

Venous puncture was performed in the operating room with an 18 or $16 \mathrm{G}$ catheter in an upper limb vein, followed by lactated Ringer's infusion $(500 \mathrm{ml})$. Monitoring included a cardioscope in $D_{\|}$lead, pulse oximetry and non-invasive automatic blood pressure. Before epidural block, patients received mild intravenous sedation with titrated fractions of midazolam $(5 \mathrm{mg})$ and fentanyl $(50 \mu \mathrm{g})$ in sufficient doses to keep patients relaxed and cooperative. Then, lumbar epidural puncture was performed with a 16G Tuohy needle. After a negative test dose with $2 \%$ lidocaine with epinephrine $1: 200,000$ (60 mg), $0.5 \%$ bupivacaine $(100 \mathrm{mg})$ with epinephrine $1: 200,000$ plus $100 \mu \mathrm{g}$ fentanyl were injected. Next, a catheter was inserted in the epidural space in the cephalad direction aiming at postoperative patient controlled analgesia with an infusion pump, which was connected to the epidural catheter after surgery.

In the immediate postoperative period, patients were randomly distributed in two groups: Group $F(n=35)$ and Group S $(n=35)$, which received solutions and schedules shown in tables I and II.

Table I - Group F - Fentanyl-Containing Solution ${ }^{8}$

\begin{tabular}{ll}
\hline Solution Composition & \\
Saline solution & $85 \mathrm{ml}$ \\
$0.5 \%$ bupivacaine & $5 \mathrm{ml}(25 \mathrm{mg})$ \\
Fentanyl & $10 \mathrm{ml}(500 \mu \mathrm{g})$ \\
Infusion schedule & \\
Continuous infusion & $5 \mathrm{ml} . \mathrm{h}^{-1}$ \\
Bolus release & $2 \mathrm{ml}$, at 15-minute intervals \\
\hline
\end{tabular}


Table II - Group S - Sufentanil-Containing Solution

\begin{tabular}{ll}
\hline $\begin{array}{l}\text { Solution Composition } \\
\text { Saline solution }\end{array}$ & $92 \mathrm{ml}$ \\
$0.5 \%$ bupivacaine & $5 \mathrm{ml}(25 \mathrm{mg})$ \\
Sufentanil & $3 \mathrm{ml}(150 \mu \mathrm{g})$ \\
Infusion schedule & \\
Continuous infusion & $5 \mathrm{ml} \cdot \mathrm{h}^{-1}$ \\
Bolus release & $2 \mathrm{ml}$, at 15 -minute intervals \\
\hline
\end{tabular}

If more or less analgesia was required, infusion rate was up or down adjusted in $20 \%$ of the initial dose. In the presence of undesirable side effects, doses would also be reset to less $20 \%$ of previous ones. In the persistence of undesirable effects, they would be treated as follows: for severe pruritus -1 ) muscular diphenydramine (50 mg);2) $2 \mathrm{ml}$ naloxone $+8 \mathrm{ml}$ distilled water intravenously titrated. Respiratory depression with respiratory rate equal to or less than $9 \mathrm{ipm}$ would be treated with the same kind of naloxone administration. Intravenous metoclopramide (10 $\mathrm{mg}$ ) would be administered for nausea and vomiting.

Sedation was evaluated through the following parameters: 0 = no sedation; 1 = mild sedation (occasionally sleepy, awakens when called); 2 = moderate sedation (often sleepy, awakens when called); 3 = deep sedation (sleepy, awakens with painful stimulus). Bromage's scale was used to evaluate motor block: $0=$ no motor block; $1=$ unable to raise stretched legs; 2 = unable to bend knees; 3 = unable to bend feet.

Analgesia was evaluated through the 0 to 10 Analog Visual Scale (AVS) where 0 means no pain and 10 the worst possible pain. Analgesia was evaluated in two periods: 12 hours after surgery and then until patients discharge. The following parameters were measured: total infused solution volume, total opioid dose, infusion time, number of requested boluses and number of released boluses. Side-effects, such as nausea, vomiting, pruritus, urine retention, sedation and respiratory depression were recorded.

Student's $t$ test for Two Independent Groups was used for statistical analysis of age, height, weight, nausea, vomiting, total epidural infusion volume, number of requested boluses, number of released boluses and infusion time. Mann-Whitney non-parametric test was used for statistical analysis of physical status (ASA), gender, pruritus, sedation, urine retention and pain Analog Visual Scale (AVS) scores. Significance level was established to $5 \%(p<0.05)$.

\section{RESULTS}

There were no statistically significant differences in age, weight, height, gender and physical status between groups (Table III). There was no statistical evidence that treatments were different regarding pain intensity rated by the Analog Visual Scale (AVS) during the first 12 postoperative hours and, in the subsequent hours until patients' discharge. The same is true for the number of released boluses. However, a signifi- cant difference was seen in the number of bolus requests: Group F (fentanyl) has requested more boluses (Table IV).

Table III - Demographics Data

\begin{tabular}{|c|c|c|c|c|c|}
\hline & $\begin{array}{c}\text { Age * } \\
\text { (years) }\end{array}$ & $\begin{array}{c}\text { Weight * } \\
(\mathrm{kg})\end{array}$ & $\begin{array}{l}\text { Height * } \\
(\mathrm{cm})\end{array}$ & $\begin{array}{c}\text { Gender } \\
(\mathrm{M} / \mathrm{F})\end{array}$ & $\begin{array}{c}\text { ASA } \\
1 / I I\end{array}$ \\
\hline $\begin{array}{l}\text { Group F } \\
(n=35)\end{array}$ & $28.40 \pm 8.74$ & $79.77 \pm 14.84$ & $175.49 \pm 8.05$ & $33 / 2$ & $26 / 9$ \\
\hline $\begin{array}{l}\text { Group S } \\
(\mathrm{n}=35)\end{array}$ & $28.51 \pm 7.69$ & $81.85 \pm 12.21$ & $178.91 \pm 6.95$ & $33 / 2$ & $30 / 5$ \\
\hline
\end{tabular}

Table IV - Number of Requested Boluses, Number of Released Boluses, Total Infused Volume and Mean Infusion Time (Mean \pm SD)

\begin{tabular}{lcc}
\hline Events & $\begin{array}{c}\text { Group } \mathrm{F} \\
(\mathrm{n}=35)\end{array}$ & $\begin{array}{c}\text { Group S } \\
(\mathrm{n}=35)\end{array}$ \\
\hline $\mathrm{N}^{\circ}$ of requested boluses & $7.77 \pm 7.10^{*}$ & $4.51 \pm 4.93^{*}$ \\
$\mathrm{~N}^{\circ}$ of released boluses & $4.54 \pm 2.94$ & $3.09 \pm 2.93$ \\
Total infusion volume $(\mathrm{ml})$ & $78.97 \pm 17.35$ & $87.00 \pm 18.64$ \\
Infusion time (hour) & $17.10 \pm 0.16$ & $18.25 \pm 0.16$ \\
\hline
\end{tabular}

* Statistical difference $(p<0.05)$

Considering the scores 0 (no pain), 1 and 2 (mild-tolerable pain), there has been a good response to postoperative pain treatment in both groups (Tables $\mathrm{V}$ and $\mathrm{VI}$ ). There was no case of unbearable pain in any group, and only 2 cases in Group F where pain reached score 8. Urine retention was seen in 5 sufentanil group patients, demanding a vesical catheter placement (Table VII).

Sedation was scored zero for most patients (34 patients in each group) with no significant difference. Two mild sedation cases were recorded ( 1 in each group) (Table VIII). Pruritus had a similar behavior, requiring no treatment (Table IX).

Although low, the incidence of vomiting was higher in sufentanil group (Table X).

Table V - Analog Visual Scale (AVS) Applied as from the $13^{\text {th }}$ Hour until Patients' Discharge

\begin{tabular}{lcc}
\hline Scores & $\begin{array}{c}\text { Group F } \\
(\mathrm{n}=35)\end{array}$ & $\begin{array}{c}\text { Group S } \\
(\mathrm{n}=35)\end{array}$ \\
\hline 0 & 25 & 17 \\
1 & 5 & 7 \\
2 & 3 & 2 \\
3 & 0 & 4 \\
4 & 1 & 1 \\
5 & 1 & 2 \\
6 & 0 & 0 \\
7 & 0 & 2 \\
8 & 0 & 0 \\
9 & 0 & 0 \\
10 & 0 & 0 \\
\hline
\end{tabular}


Table VI - Analog Visual Scale (AVS) Applied During the First 12 Postoperative Hours

\begin{tabular}{lcc}
\hline Scores & $\begin{array}{c}\text { Group } F \\
(n=35)\end{array}$ & $\begin{array}{c}\text { Group S } \\
(n=35)\end{array}$ \\
\hline 0 & 11 & 13 \\
1 & 8 & 6 \\
2 & 7 & 4 \\
3 & 2 & 5 \\
4 & 1 & 3 \\
5 & 3 & 4 \\
6 & 0 & 0 \\
7 & 1 & 0 \\
8 & 2 & 0 \\
9 & 0 & 0 \\
10 & 0 & 0 \\
\hline
\end{tabular}

Table VII - Need for Postoperative Vesical Catheter

\begin{tabular}{lcc}
\hline$N^{\circ}$ of catheters & $\begin{array}{c}\text { Group } F \\
(\mathrm{n}=35)\end{array}$ & $\begin{array}{c}\text { Group } S \\
(\mathrm{n}=35)\end{array}$ \\
\hline 0 & 35 & 30 \\
1 & 0 & 5 \\
\hline
\end{tabular}

Table VIII - Sedation

\begin{tabular}{lcc}
\hline Score & $\begin{array}{c}\text { Group } F \\
(\mathrm{n}=35)\end{array}$ & $\begin{array}{c}\text { Group S } \\
(\mathrm{n}=35)\end{array}$ \\
\hline 0 & 34 & 34 \\
1 & 1 & 1 \\
2 & 0 & 0 \\
\hline
\end{tabular}

Table IX - Pruritus

\begin{tabular}{lcc}
\hline Score & $\begin{array}{c}\text { Group F } \\
(\mathrm{n}=35)\end{array}$ & $\begin{array}{c}\text { Group S } \\
(\mathrm{n}=35)\end{array}$ \\
\hline 0 (absent) & 34 & 34 \\
1 (light) & 1 & 1 \\
2 (moderate) & 0 & 0 \\
3 (severe) & 0 & 0 \\
\hline
\end{tabular}

Table X - Nausea and Vomiting During Infusions

\begin{tabular}{lcccc}
\hline Groups & GF & GF $\%$ & GS & GS \% \\
\hline Nausea & 2 & 5.71 & 3 & 8.57 \\
Vomiting & $1^{*}$ & $2.85^{*}$ & $2^{*}$ & $5.71^{*}$ \\
\hline
\end{tabular}

*Statistical difference

No motor block was observed during infusions after lower limbs movement recovery in the PACU.

\section{DISCUSSION}

Knee ligament reconstruction is highly prevalent among young males and its postoperative period is very painful, especially during the first 24 hours when the operated knee is initially moved aiming early physiotherapy.
According to some authors ${ }^{1,8,25-28}$, epidural anesthesia with long-lasting local anesthetics associated to opioids and an epidural catheter for postoperative analgesia is the best choice for such procedure. We have used $0.5 \%$ bupivacaine with epinephrine 1:200,000 associated to fentanyl $(100 \mu \mathrm{g})$ to promote intraoperative analgesia. This fentanyl dose used in the beginning of epidural anesthesia has not interfered with the results of postoperative analgesia due to its short-duration effect.

PCA pump was installed before anesthetic blockade recovery with patients still in the operating room. Patients' discharge from PACU, however, was only authorized after total motor block recovery.

Fentanyl is a lipophilic drug with higher analgesic potency than morphine, which is hydrophilic. Some authors, however, have shown an analgesic similarity between both drugs ${ }^{2,6,8}$, but morphine showing more side-effects (nausea, vomiting and sedation). In our study, the comparison between epidural fentanyl and sufentanil associated to low concentration bupivacaine in PCA has shown efficient postoperative analgesia, with a minimum of side-effects statistical analysis of Analog Visual Scale results has shown no difference between groups in the treatment of postoperative pain until patients' discharge. However, two fentanyl group patients presented with AVS score 8 during movement, demanding a $20 \%$ increase in infusion rate. The number of released boluses, total volume and infusion time presented no significant differences, showing a similar analgesic efficacy of fentanyl and sufentanil in treating postoperative pain.

With low bupivacaine concentration $(0.025 \%)$, we had no motor block case, showing that higher bupivacaine concentrations are needed for that. Patients were kept totally awaken in the postoperative period with only 1 patient in each group with score 1 in the sedation scale. No respiratory depression was seen, showing that patients were protected against this fearful side-effect with those doses of both opioids. Few patients had nausea or vomiting in both groups $(G F=3$ and $G S=5)$, with statistically significant difference favoring fentanyl. Just one patient in each group referred mild pruritus. Urine retention was seen in 5 sufentanil group patients demanding a vesical catheter. No case was observed in fentanyl group.

Analgesia quality and the low incidence of complications have shown that both fentanyl and sufentanil are better than morphine for PCA postoperative analgesia in patients submitted to knee ligament reconstruction. This is ratified by a different study comparing morphine and fentanyl with the same method ${ }^{8}$.

The conclusion was that, in the conditions of this study, postoperative analgesia with opioids continuous epidural infusion associated to patient controlled boluses through a PCA pump is an excellent method for treating pain in patients submitted to knee ligament reconstruction, especially when low concentration of long lasting local anesthetics are associated to opioids such as fentanyl and sufentanil. Both fentanyl and sufentanil were effective in promoting analgesia. How- 
ever, sufentanil had slightly more intense side-effects as compared to fentanyl.

\section{REFERÊNCIAS - REFERENCES}

01. Braz JRC, Vanni SMD, Menezes JA et al - Associação de opióides lipofílicos à bupivacaína na anestesia peridural. Há vantagem no aumento da dose de opióide? Rev Bras Anestesiol,1998;48:455-467.

02. de Leon-Casasola AO, Lema MJ - Postoperative epidural opioid analgesia: What are the choices? Anesth Analg, 1996;83: 867-875.

03. Liu SS, Allen HW, Olsson GL - Patient-controlled epidural analgesia whit bupivacaine and fentanyl on hospital wards: prospective experience whit 1030 surgical patients. Anesthesiology, 1998;88:688-695

04. Tanaka M, Watanabe S, Ashimura $\mathrm{H}$ et al - Minimum effective combination dose of epidural morphine and fentanyl for posthysterectomy analgesia: a randomized, prospective, double-blind study. Anesth Analg, 1993;77:942-946.

05. Coda BA, Brown MC, Schaffer R et al - Pharmacology of epidural fentanyl, alfentanyl and sufentanyl in volunteers. Anesthesiology, 1994;81:1149-1161.

06. Boudreault D, Brasseva L, Samii J et al - Comparison of continuous epidural infusion or patient-controlled epidural injection of fentanyl for postoperative analgesia. Anesth Analg, 1991;73: 132-137.

07. de Leon-Casasola AO, Parker BM, Lema MJ et al - Epidural analgesia versus intravenous patient-controlled analgesia. Differences in the postoperative course of cancer patients. Reg Anesth, 1994;19:307-315.

08 - Lutti MN, Simoni RF, Cangiani LM et al - Analgesia controlada pelo paciente com morfina ou fentanil no pós-operatório de reconstrução de ligamentos do joelho: estudo comparativo. Rev Bras Anestesiol, 2000;50:8-13.

09. Lubenow JR, Janck EN, Hopkin EM et al - Comparison of patient-assisted epidural analgesia with continuous infusion epidural analgesia for postoperative patients. Reg Anesth, 1994;19:206-211.

10. Camu F, Van Aken H, Bovill JG - Postoperative analgesic effects of three demand-dose sizes of fentanyl administered by patient-controlled analgesia. Anesth Analg, 1998;87: 890-895.

11. Niemi G, Breivik H - Adrenaline markedly improves thoracic epidural analgesia produced by a low-dose infusion of bupivacaine, fentanyl and adrenaline after major surgery. A randomized, double-blind, cross-over study with and without adrenaline. Acta Anaesthesiol Scand, 1998;42:897-909.

12. Sjÿostrÿom $\mathrm{S}$, Blÿass $\mathrm{J}$ - Postoperative analgesia with epidural bupivacaine and low-dose fentanyl - a comparison of two concentrations. Acta Anaesthesiol Scand, 1998;42:776-782.

13. Berti M, Fanelli G, Casati A et al - Patient supplemented epidural analgesia after major abdominal surgery with bupivacaine/ fentanyl or ropivacaine/ fentanyl. Can J Anaesth, 2000;47: 27-32.

14. Silvasti M, Pitkÿanen M - Continuous epidural analgesia with bupivacaine-fentanyl versus patient-controlled analgesia with i.v. morphine for postoperative pain relief after knee ligament surgery. Acta Anaesthesiol Scand. 2000;44:37-42.

15. Chrubasik S, Chrubasik J, Pfisterer M et al - Comparison of morphine with and without fentanyl for epidural analgesia after major abdominal surgery. Reg Anesth, 1996;21:175-181.
16. Boudreault D, Brasseva L, Samii K et al - Comparison of continuous epidural infusion or patient-controlled epidural injection of fentanyl for postoperative analgesia. Anesth Analg, 1991;73:132-137.

17. Dahl JB, Rosenberg J, Hansen BL et al - Differential analgesic effects of low-dose epidural morphine and morphinebupivacaine at rest and during mobilization after major abdominal surgery. Anesth Analg, 1992;74:362-365

18. Connelly NR, Parker RK, Vallurupalli V et al - Comparison of epidural fentanyl versus epidural sufentanil for analgesia in ambulatory patients in early labor. Anesth Analg, 2000;91: 374-378.

19. Vercauteren MP, Van Den Bergh L, Kartawiadi SL et al - Addition of bupivacaine to sufentanil in patient-controlled epidural analgesia after lower limb surgery in young adults: effect on analgesia and micturition. Reg Anesth Pain Med, 1998;23:182-188.

20. Kopacz DJ, Sharrock NE, Allen HW - Comparison of levobupivacaine $0.125 \%$, fentanyl $4 \mathrm{microg} / \mathrm{ml}$, ou their combination for patient-controlled epidural analgesia after major orthopedic surgery. Anesth Analg, 1999;89:1497-1503.

21. Danou F, Paraskeva A, Vassilakopoulos T et al - The analgesic efficacy of intravenous tenoxicam as an adjunct to patient-controlled analgesia in total abdominal hysterectomy. Anesth Analg, 2000;90:672-676.

22. Shiihara K, Kohno K, Kosaka Y - Postoperative epidural analgesia after upper abdominal surgery: the effects of low concentrations of bupivacaine combined with a low dose of opioid. Masui, 1999;48:731-738.

23. Mahon SV, Berry PD, Jackson M et al - Thoracic epidural infusions for post-thoracotomy pain: a comparison of fentanylbupivacaine mixtures vs. Fentanyl alone. Anaesthesia, 1999; 54:641-646.

24. Colbert S, O'Hanlon DM, Chambers F et al - The effect of intravenous tenoxicam on pruritus in patients receiving epidural fentanyl. Anaesthesia. 1999;54:76-80.

25. Broekema AA, Veen A, Fidler V et al - Postoperative analgesia with intramuscular morphine at fixed rate versus epidural morphine or sufentanil and bupivacaine in patients undergoing major abdominal surgery. Anesth Analg, 1998;87: 1346-1353

26. Komatsu H, Matsumoto S, Mitsuhata $\mathrm{H}$ et al - Comparison of patient-Controlled epidural analgesia with and without background infusion after gastrectomy. Anesth Analg, 1998;87: 907-910.

27. Duval Neto GF - Bloqueio peridural: controle de qualidade. Rev Bras Anestesiol, 1997;47:543-546.28. Kampe S, Weigand C, Kaufmann $\mathrm{J}$ et al - Postoperative analgesia with no motor block by continuos epidural infusion of ropivacaine $0.1 \%$ and sufentanil after total hip replacement. Anesth Analg, 1999;89: 395-398.

\section{RESUMEN}

Lutti MN, Vieira JL, Eickhoff DR, Carli D, Carvalho MA - Analgesia Controlada por el Paciente con Fentanil o Sufentanil en el Pós-Operatorio de Reconstrucción de Ligamentos de la Rodilla: Estudio Comparativo

Justificativa y Objetivos - Los opioides han sido utilizados por vía peridural asociados o no a anestésicos locales para analgesia pós-operatoria de forma continua y/o en bolus controlado por el paciente. El objetivo de este estudio fue comparar la analgesia pós-operatoria entre el fentanil y sufentanil en infusión continua y en bolus por vía peridural, en 
pacientes sometidos a la reconstrucción de ligamento de la rodilla.

Método - Participaron del estudio 70 pacientes con edad entre 16 y 47 anos, estado físico ASA I y II, divididos aleatoriamente en dos grupos: Grupo F (fentanil) y Grupo S (sufentanil). Todos los pacientes fueron sometidos a anestesia peridural con bupivacaína a 0,5\% (100 mg) con epinefrina 1:200.000 asociada a fentanil $(100 \mathrm{mg})$. Al final de la cirugía, los pacientes recibieron fentanil (Grupo F) o sufentanil (Grupo S) por vía peridural en régimen de infusión continua más bolus liberados por el paciente. En el Grupo F fue utilizada solución fisiológica $(85 \mathrm{ml})$ conteniendo fentanil $500 \mu \mathrm{g}(10 \mathrm{ml})$ y bupivacaína $(5 \mathrm{ml}$ a $0,5 \%)$. En el Grupo S fue utilizada solución fisiológica (92 ml) conteniendo sufentanil $150 \mu \mathrm{g}(3 \mathrm{ml})$ y bupivacaína $(5 \mathrm{ml} \mathrm{a}$ $0,5 \%)$. Para los dos grupos la bomba de infusión fue programada inicialmente en $5 \mathrm{ml} . \mathrm{h}^{-1}$, con dosis de $2 \mathrm{ml}$ en bolus liberado por el paciente en un intervalo de 15 minutos. Fueron comparados los siguientes parámetros: dolor, número de bolus accionados, consumo de opioides, bloqueo motor, sedación y efectos colaterales.

Resultados - No hubo diferencia significativa entre los grupos cuanto la calidad de la analgesia, siendo la mayoría de buena calidad (EAV $O$ a 2). Hubo diferencia significativa cuanto al número de bolus liberados. En el Grupo $F$ fue mayor que el Grupo S. No hubo diferencia cuanto al volumen total de la solución infundida y tiempo de infusión total. No hubo bloqueo motor después de la institución de la analgesia controlada por el paciente $(A C P)$. La incidencia de vómitos y retención urinaria fue mayor en el Grupo $S$ y cuanto a la sedación y al prurito, no hubo diferencia entre los grupos.

Conclusiones - El fentanil y el sufentanil continuos y en bolus accionados por el paciente, por vía peridural, en las dosis utilizadas en este estudio, presentaron excelente analgesia pós-operatoria. No obstante, el sufentanil presentó efectos colaterales mas intensos que el fentanil. 